\title{
VirES for Aeolus
}

Virtual Workspaces for Earth Observation Scientists
Specification, Development, Maintenance \& Pre-Operations

VirES for Aeolus

Online visual analysis of Aeolus data

EGU2020-22439

Session AS1.35

05 May 2020, 08:30-10:15

Daniel Santillan, Christian Schiller, Markus Meringer, Oliver Reitebuch, Fabian Weiler, Dorit Huber 


\section{Outlook}

() Presentation of service

- Overview of webclient

( Examples of available products

- L1B, L2A, L2B, L2C

- AUX MRC, RRC, ISR, ZWC, MET

○ ADAM Albedo

() Conclusion 


\section{VirES}

\section{Aeolus}

\section{VirES for Aeolus Service}

(-) Web client accessible with any modern browser to directly visualize and interact with ESA's Earth Explorer Aeolus wind profiling mission

\section{https://aeolus.services}

- Service provided by ESA, implemented and operated by EOX

(- Highly interactive data exploitation and retrieval interface

- Collaboration between experts in the field and project partners DLR and DoRIT and service developer EOX to understand and provide functions relevant to users

(- Service has been actively further developed and improved since the Aeolus launch August 2018 in close collaboration with its users and project partners

- Currently made available for Cal/Val users, will be open to the public when the data is made available publicly 


\section{VirES}

Aeolus

\section{Webclient overview}

( Workflow:

- Interactive tutorial available to quickly get you introduced to the client features

- Selection of desired product

- Selection of time of interest

- Timeline shows data availability

- Analysis of displayed data on 3D globe and on analytics panel

- Possibility to download selected data for further processing (netCDF and packaged original files)

(- 3D globe:

- Fully interactive 3D visualization

- Provides 3 different projections (3D globe, 2.5D "columbus" and 2D)

- Selection and styling of parameter to be shown in products settings

- Possibility to import $\mathrm{kml}$ to display additional features

- Analytics panel:

- Fully interactive plotting widget

- Axis independent zoom, pan, information on every rendered point, and more

- Customizable plot configuration

- Any number of connected plots

- Axis configuration

- Extensive parameter configuration options

- Interactive filters panel with histogram information on data distribution 


\section{Aeolus}

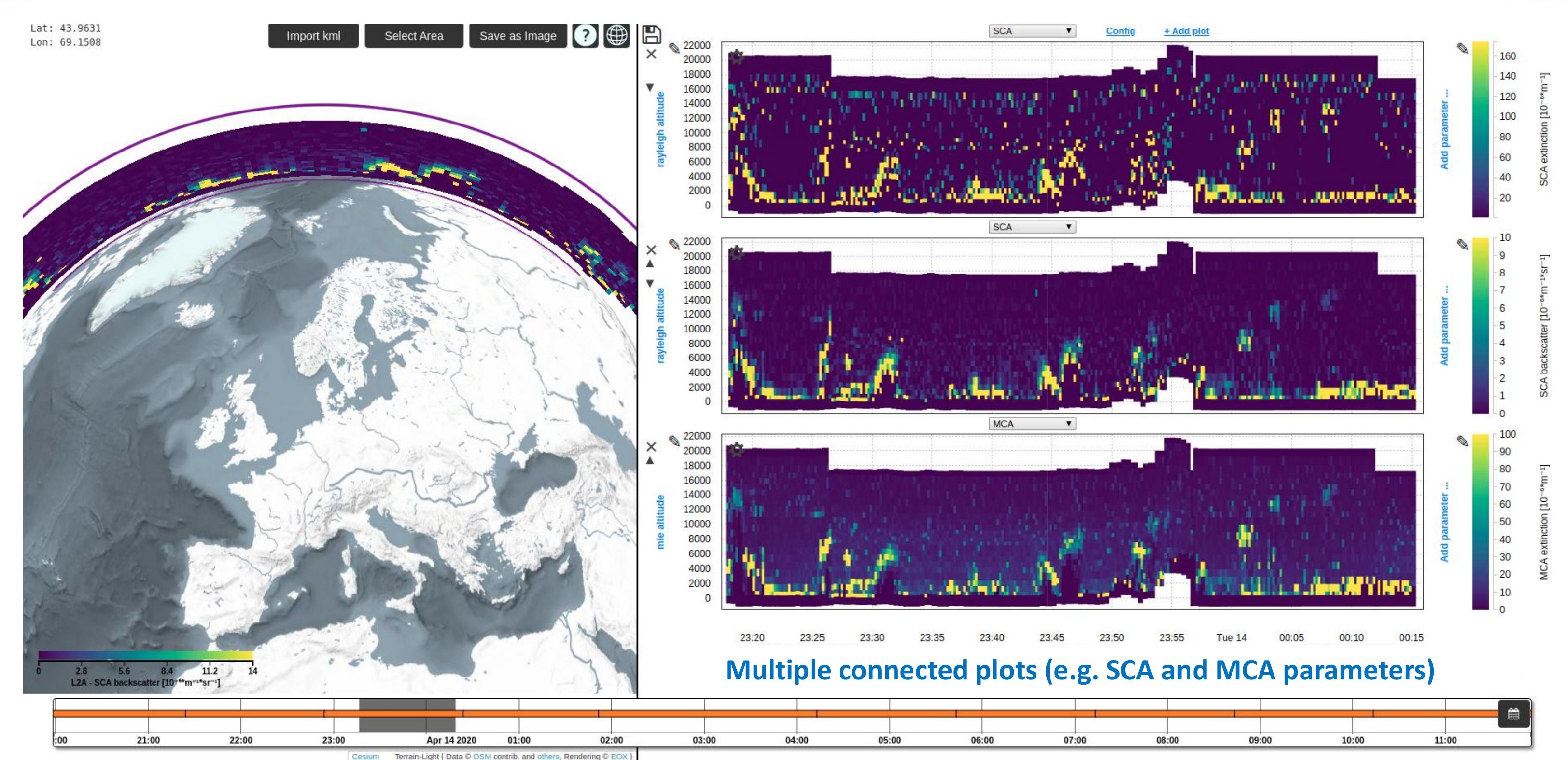




\section{Aeolus}

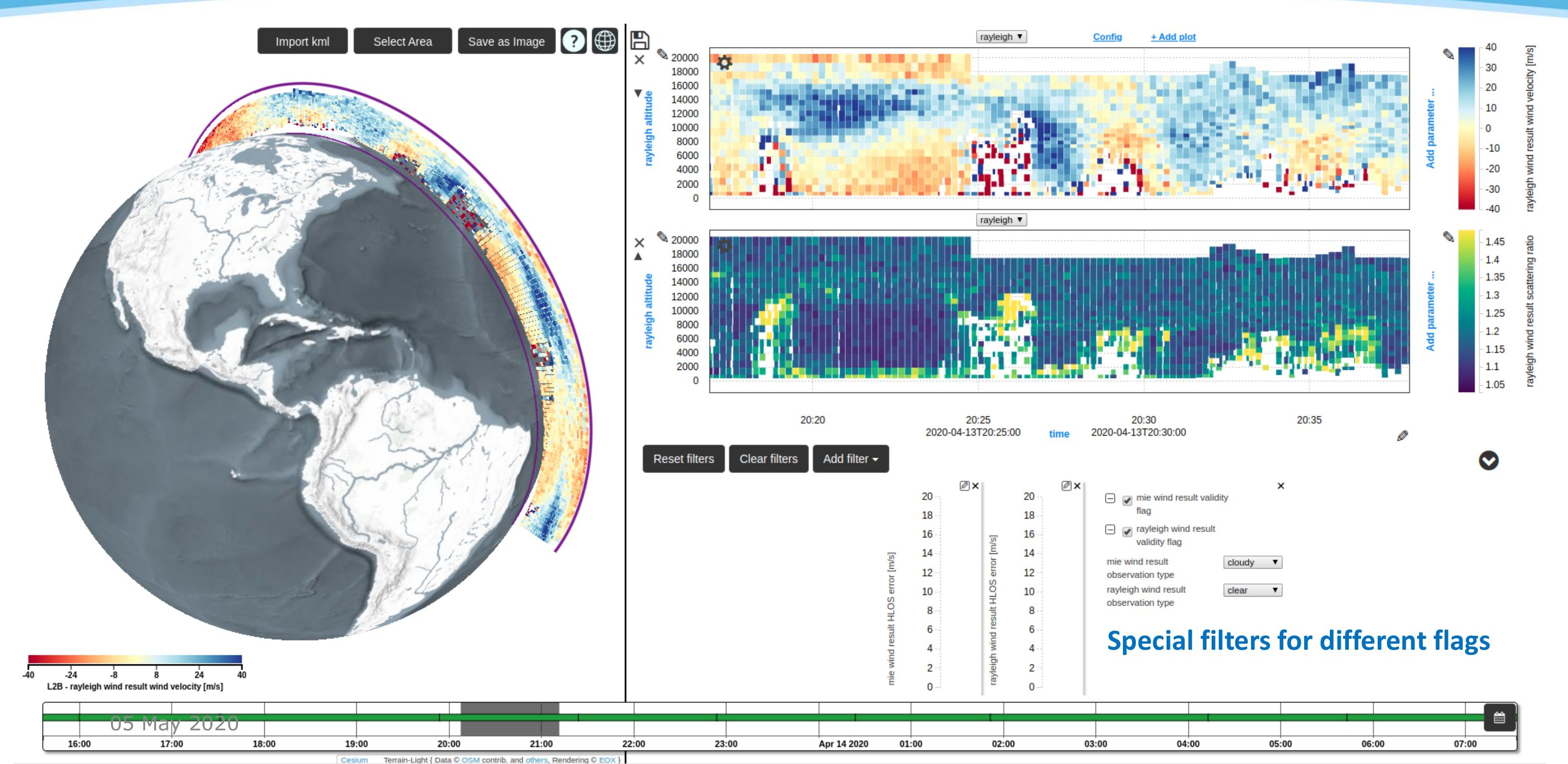




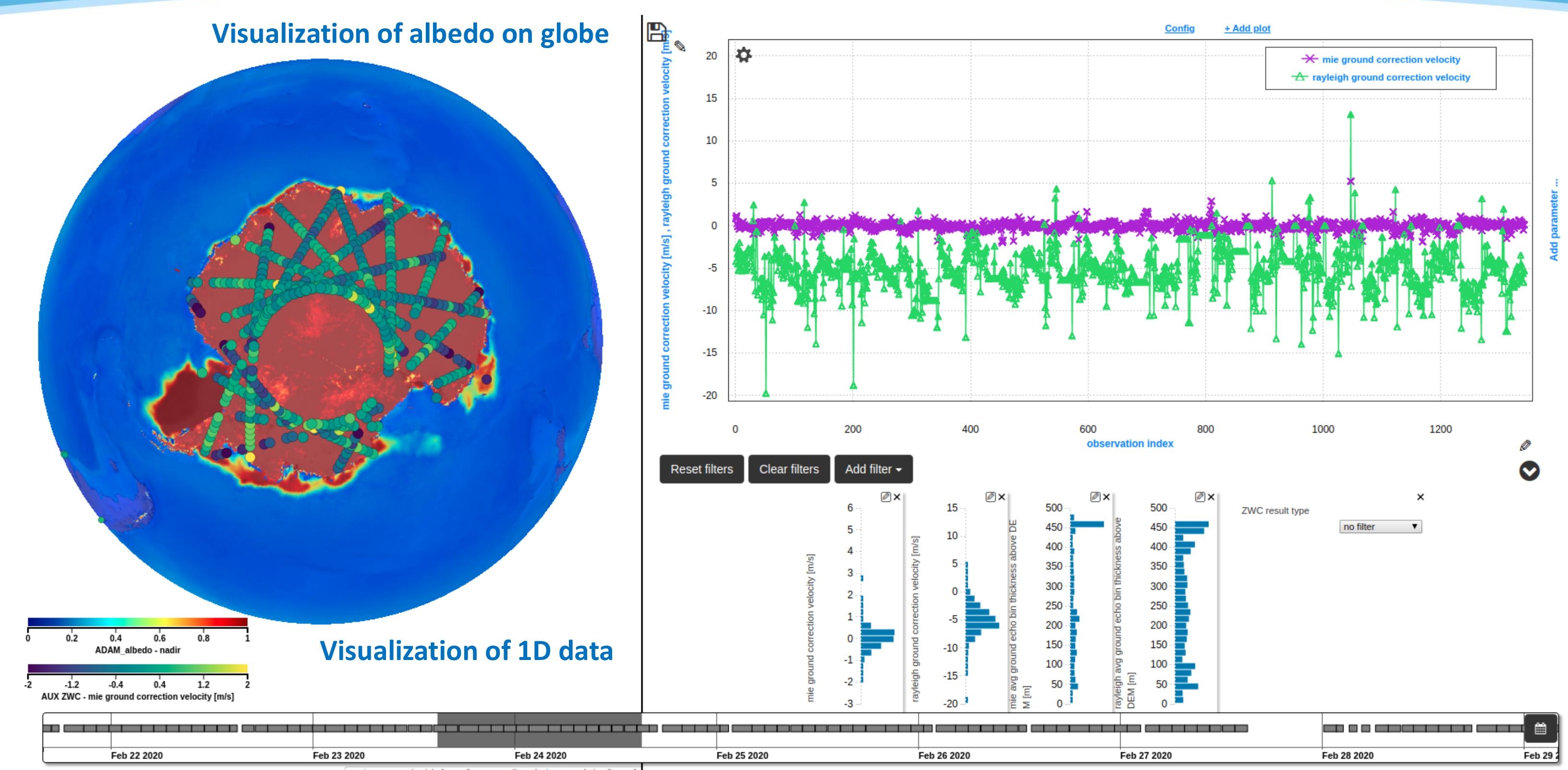


Aeolus

Visualization of 1D on plots, customization of symbol, colour, line connection, ...
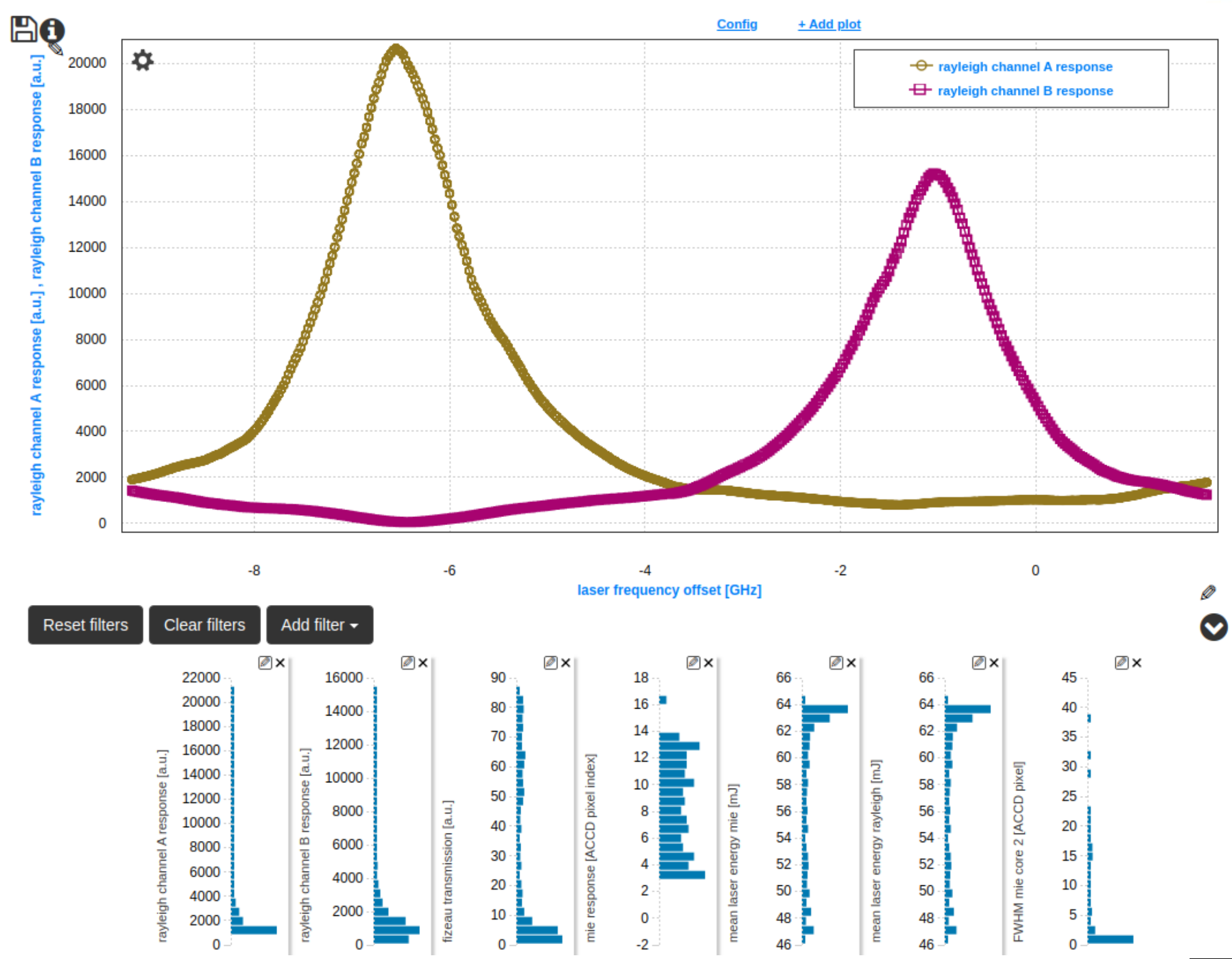


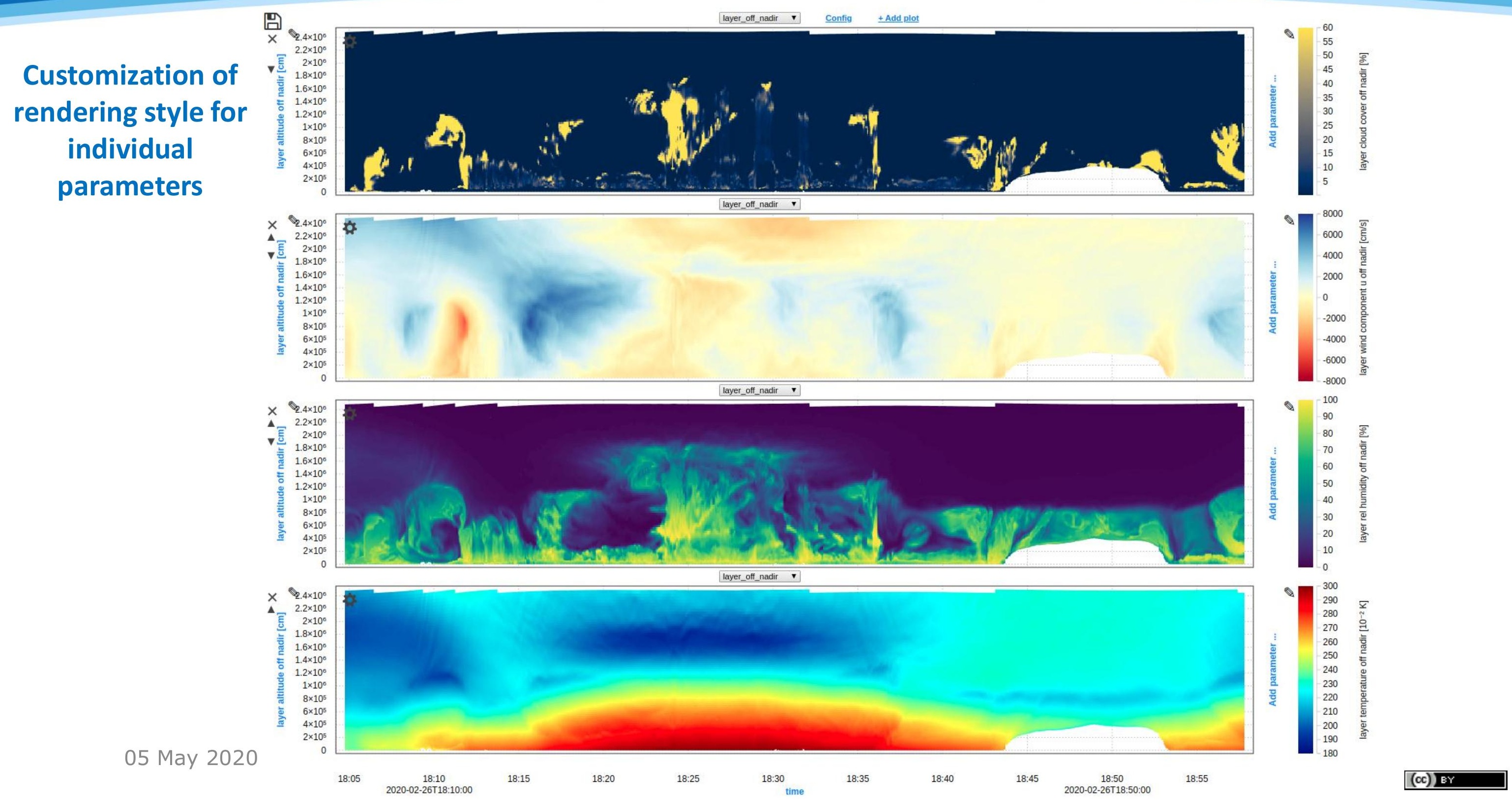




\section{Conclusion}

- VirES for Aeolus will be made available to the general public synchronized with the NRT wind data release planned on the 12 May 2020

Web service access currently only granted by ESA (Contact EO Help desk to request account)

(-) Service in continuous development, all feedback, questions and feature suggestions are welcome!

\section{https://aeolus.services}

\section{Contact}

feedback@vires.services

daniel.santillan@eox.at 